\title{
Diffusion of Water Molecules in Amorphous Silica
}

\author{
Sarah Kostinski, Ravindra Pandey, S. Gowtham, Udo Pernisz, and Alexander Kostinski
}

\begin{abstract}
The diffusive penetration of atmospheric water vapor into amorphous silica $\left(\mathrm{a}-\mathrm{SiO}_{2}\right)$ degrades the performance of electronic devices. In this letter, we calculate the range of activation energies for water diffusion in $\mathrm{a}_{-} \mathrm{SiO}_{2}$ such that the diffusion time through, for example, a $0.5-\mu \mathrm{m}$ protective layer is on the order of the decadal time scale, as required in typical applications. We find that for all practical purposes, silica composed of $n$-member rings is impenetrable to water vapor for $n \leq 5$. Thus, we conclude that the distribution of $n$-member rings in $\mathrm{a}^{-} \mathrm{SiO}_{2}$ and, specifically, the $n>5$ fraction is the critical parameter for predicting device performance.
\end{abstract}

Index Terms-Amorphous silica $\left(\mathrm{a}-\mathrm{SiO}_{2}\right)$, diffusion, thin film, water vapor.

\section{INTRODUCTION}

A MORPHOUS silica $\left(\mathrm{a}-\mathrm{SiO}_{2}\right)$ is ubiquitous in electronic devices. Since the devices are exposed to water vapor in ambient conditions, the performance of devices coated with $\mathrm{a}-\mathrm{SiO}_{2}$ protective thin films is limited by water-vapor diffusive penetration [1], causing, for example, puzzling film corrosion [2]. Therefore, it is desirable to render such silica films impermeable to water-vapor molecules.

a- $\mathrm{SiO}_{2}$ has a network structure characterized by rings of $n$ members (i.e., silicon atoms), where $n$ typically ranges from three to nine with $n=6$ being the most frequently occurring ring size [3], [4]. The reactions of water molecules with such silica ring networks were recently investigated in [5], while activation energy calculations for the diffusion of water in silicon carbide $(\mathrm{SiC})$ were presented in [6]. Here, we address the "impermeable-design" problem via diffusive scaling combined with the calculations of ring barrier energies based on first-principles methods. Specifically, we ask the following questions: 1) What is the range of activation (barrier) energies such that the diffusion time through, for example, a $0.5-\mu \mathrm{m}$ protective layer is about several years (as required in typical applications)?, and 2) how sensitive is the diffusion time to changes in ring-energy-barrier estimates? Both $\mathrm{a}-\mathrm{SiO}_{2}$ and amorphous silicon carbide (a-SiC) are considered as they share

Manuscript received February 1, 2012; revised February 24, 2012; accepted February 25, 2012. Date of publication April 4, 2012; date of current version May 18, 2012. This work was supported in part by National Science Foundation (NFS) under Grant AGS-1119164 and in part by an NSF Graduate Research Fellowship under Grant DGE-1144152. The review of this letter was arranged by Editor S. List.

S. Kostinski is with Harvard University, Cambridge, MA 02138 USA (e-mail: skostinski@fas.harvard.edu).

R. Pandey, S. Gowtham, and A. Kostinski are with Michigan Technological University, Houghton, MI 49931 USA (e-mail: pandey@mtu.edu; sgowtham@mtu.edu; kostinsk@mtu.edu).

U. Pernisz is with Dow Corning Corporation, Midland, MI 48686 USA (e-mail: upernisz@earthlink.net).

Color versions of one or more of the figures in this letter are available online at http://ieeexplore.ieee.org.

Digital Object Identifier 10.1109/LED.2012.2189750 the ring-based network structure and industrial applications as protective coatings [6].

\section{Diffusive Scaling Estimates}

The mean diffusion time through a distance $L$ is given by

$$
t \approx \frac{L^{2}}{D}
$$

where $D$ is the diffusion coefficient, $t$ is the mean (averaged over the molecules) or characteristic time scale, and $L$ is the desired diffusion distance [7].

The diffusion coefficient is

$$
D=D_{0} e^{-E_{a} / k T}
$$

where the prefactor $D_{0}=a^{2} \nu z / 6$, with $a$ as the distance (normal to ring plane) between adjacent sites, $\nu$ as the IR vibrational frequency, and $z / 6=1$ for the geometric model of a simple ring [6].

To express the activation energy as a function of time, we substitute and rearrange as follows:

$$
t=\frac{L^{2}}{D_{0}} e^{E_{a} / k T} \rightarrow e^{E_{a} / k T}=\frac{D_{0}}{L^{2}} t \rightarrow \frac{E_{a}}{k T}=\ln (t / \tau)
$$

yielding

$$
E_{a}=k T \ln (t / \tau)
$$

where $\tau=L^{2} / D_{0}=1 / \nu(L / a)^{2}$ is the characteristic time.

To gain an intuitive feeling for the magnitudes of required barrier energies, for SiC [6], we set $a=2.7 \AA$ and $\nu=3.3 \times$ $10^{13} \mathrm{~s}^{-1}$ (taken from an IR spectrum peak near $1100 \mathrm{~cm}^{-1}$ ), yielding $D_{0}=a^{2} \nu \simeq 2.4 \times 10^{-2} \mathrm{~cm}^{2} / \mathrm{s}$. For $\mathrm{SiO}_{2}, a=1.6 \AA$ and $\nu=3.3 \times 10^{13} \mathrm{~s}^{-1}$ (taken from an IR spectrum peak near $1100 \mathrm{~cm}^{-1}[8]$ ); hence, $D_{0}=a^{2} \nu \simeq 8.4 \times 10^{-3} \mathrm{~cm}^{2} / \mathrm{s}$. At room temperature, $E_{a}=(0.026 \mathrm{eV}) \ln (t / \tau)$, and therefore, a thickness of $L=0.5 \mu \mathrm{m}$ yields $\tau=1.0 \times 10^{-7} \mathrm{~s}=3.3 \times$ $10^{-15}$ year for $\mathrm{SiC}$ and $\tau=3.0 \times 10^{-7} \mathrm{~s}=9.4 \times 10^{-15}$ year for $\mathrm{SiO}_{2}$.

In Fig. 1, we present the required $\mathrm{SiO}_{2}$ and $\mathrm{SiC}$ activation energies for various diffusion lengths $L$ and times $t$. The activation energy required for a penetration time of five years through $\mathrm{a} \mathrm{SiO}_{2}$ layer of $0.5 \mu \mathrm{m}$ is about $0.88 \mathrm{eV}$. Note the sensitivity of diffusion time to small changes in energy: A penetration time of one year requires $0.84 \mathrm{eV}$, while two years corresponds to $0.86 \mathrm{eV}$. Recently, Lechenault et al. [2] estimated the water diffusion coefficient in silica as $D \sim 10^{-21} \mathrm{~cm}^{2} \cdot \mathrm{s}^{-1}$ at room temperature. They did so by extrapolating experimentally the results of Tomozawa and Davis [9]. This corresponds to an effective activation energy of about $1.1 \mathrm{eV}$ for $\mathrm{SiO}_{2}$. 


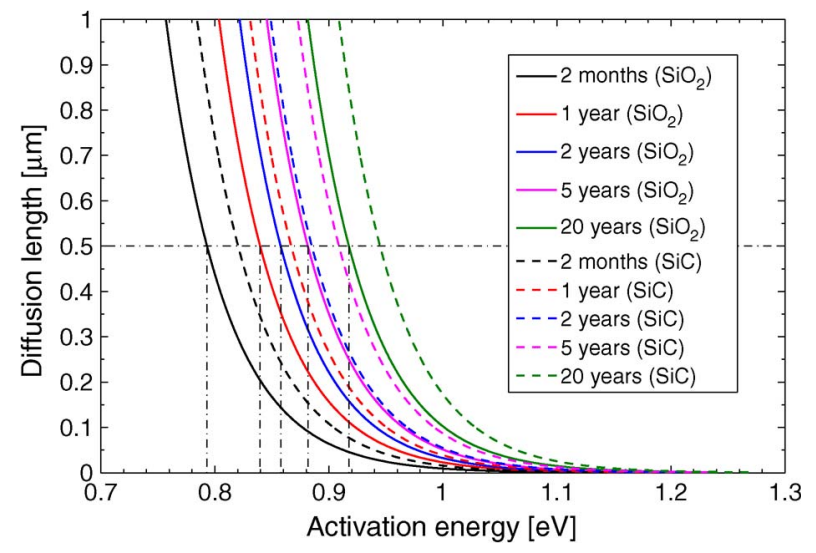

Fig. 1. Diffusion scaling versus activation energy. For example, water vapor penetration through a $0.5-\mu \mathrm{m} \mathrm{SiO}_{2}$ layer in two years corresponds to an $E_{a}$ of $0.86 \mathrm{eV}$.
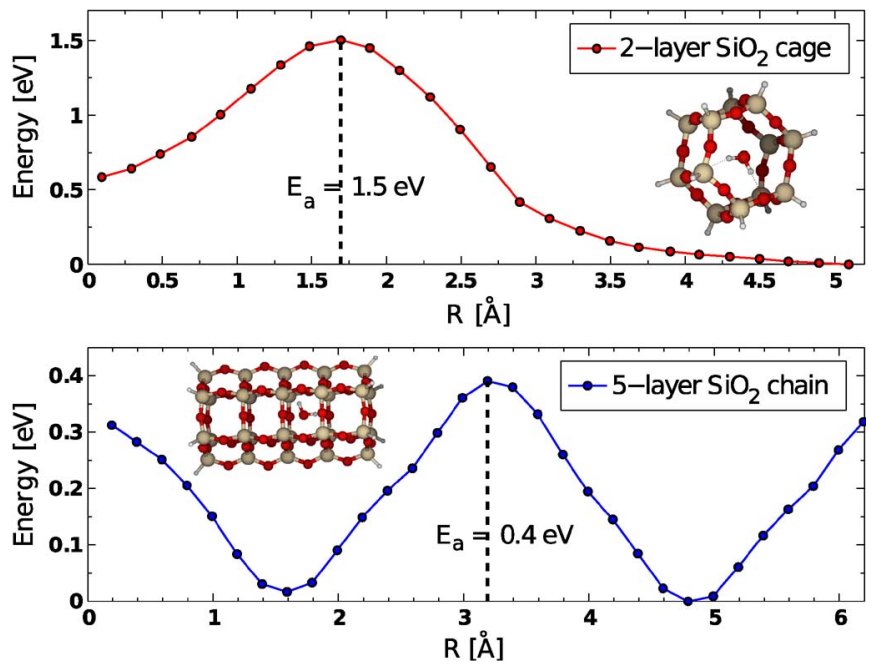

Fig. 2. Activation-energy-versus-distance results. (Top panel) Bilayer silica cage. (Bottom panel) Five-layer silica chain.

\section{Calculations}

Activation energies for water-vapor penetration and diffusion into $\mathrm{a}-\mathrm{SiO}_{2}$ and $\mathrm{a}-\mathrm{SiC}$ were calculated using the Gaussian program [10]. As in [6], the structures of the water molecule and $\mathrm{SiO}_{2}$ networks were first optimized by the Austin Model 1 (AM1) semiempirical molecular method [11] and then refined with density functional theory using the B3LYP exchange [12] and $6-31 \mathrm{G}^{*}$ basis set [13]. Good agreement with the experimental values of structural properties (within a few percent) was obtained. For example, deviations from experimental values for the water molecule were $1.3 \%$ in the $\mathrm{O}-\mathrm{H}$ bond length and $0.9 \%$ in the $\mathrm{H}-\mathrm{O}-\mathrm{H}$ bond angle.

Two variants of $\mathrm{SiO}_{2}$ networks were optimized: a bilayer cage (see inset in the top plot of Fig. 2) and a five-layer chain (see inset in the bottom plot of Fig. 2). Silicon atoms in the outermost rings were terminated by hydrogen atoms [6].

We proceeded to calculate the activation energies of water diffusion and penetration into silica ring networks using a fixed potential energy scan with the AM1 method. (As will shortly become apparent, greater accuracy in calculating barrier energies using other methods, e.g., B3LYP, is unnecessary.)
TABLE I

Bilayer $n$-MEMber Ring CAges: Activation ENERgy $\left(E_{a}\right)$ Versus Diameter $(D)$. For COMPARISON, Results FOR SiC ARE Also Given [6]

\begin{tabular}{|c|c|c|c|c|}
\hline \multirow{2}{*}{$\begin{array}{l}\text { number of } \\
\text { ring members } \\
n\end{array}$} & \multicolumn{2}{|l|}{$\mathrm{SiO}_{2}$} & \multicolumn{2}{|l|}{$\mathrm{SiC}$} \\
\hline & $D(\AA)$ & $E_{a}(\mathrm{eV})$ & $D(\AA)$ & $E_{a}(\mathrm{eV})$ \\
\hline 3 & 3.4 & 37.7 & 3.6 & 19.2 \\
\hline 4 & 4.4 & 21.0 & 4.6 & 5.1 \\
\hline 5 & 5.2 & 5.1 & 5.6 & 1.0 \\
\hline 6 & 6.4 & 1.5 & 6.6 & $\ldots$ \\
\hline 7 & 7.2 & 0.3 & $\ldots$ & $\ldots$ \\
\hline
\end{tabular}

TABLE II

Five-LAyer $n$-MEMBer Ring Chains: Activation ENERgy $\left(E_{a}\right)$ Versus Diameter $(D)$. For Comparison, SiC Results From [6] Are Also Given

\begin{tabular}{|c|c|c|c|c|}
\hline \multirow{2}{*}{$\begin{array}{l}\text { number of } \\
\text { ring members } \\
n\end{array}$} & \multicolumn{2}{|l|}{$\mathrm{SiO}_{2}$} & \multicolumn{2}{|l|}{$\mathrm{SiC}$} \\
\hline & $D(\AA)$ & $E_{a}(\mathrm{eV})$ & $D(\AA)$ & $E_{a}(\mathrm{eV})$ \\
\hline 3 & 3.4 & 41.7 & 3.5 & 9.9 \\
\hline 4 & 4.3 & 9.6 & 4.4 & 2.2 \\
\hline 5 & 5.3 & 2.0 & 5.5 & 0.4 \\
\hline 6 & 6.3 & 0.4 & 6.5 & 0.02 \\
\hline 7 & 7.2 & 0.01 & $\ldots$ & $\ldots$ \\
\hline
\end{tabular}

Unlike [5], devoted to hydrolysis reactions, no bonds were broken during the scan while determining activation energies. In the potential energy scan, the water molecule travelled along and rotated about the silica cage's central axis in order to obtain the minimum energies at each distance $R$, where $R$ is the distance between the oxygen atom of the water molecule and the center of mass of the cage structure.

\section{RESUlts AND Discussion}

Examples of the resulting energy curves versus $R$ for selected $n$ are given in Fig. 2. In the top panel are the "exterior" data of bilayer $n$-member rings (for modeling the penetration of water molecules into the outermost layer of the thin film). It is "exterior" in the sense that the energy scan began with the water molecule outside the cage which was then brought inside the cage (i.e., penetration). The bottom panel presents "interior" data, modeling a water molecule that had already penetrated and was situated inside a multilayer network (i.e., diffusion). Exterior and interior data for $\mathrm{SiC}$ and $\mathrm{SiO}_{2}$ are given in Tables I and II, respectively.

The sensitivity of diffusion times to activation energy puts these ring energies in a different perspective, as shown in Fig. 3. It can be seen from the figure that differences in activation energies for different $n$ 's are enormous, even when compared to differences in the diffusion times of two months and 20 years. Uncertainties in the estimates of $a$, e.g., 2.7 versus $1.8 \AA$, are minuscule by comparison. However, because $E_{a}$ is linear in $T$, the activation energy is sensitive to changes in environmental temperature. For example, the temperature of a microchip's surroundings may increase as a computer heats up, and for a 


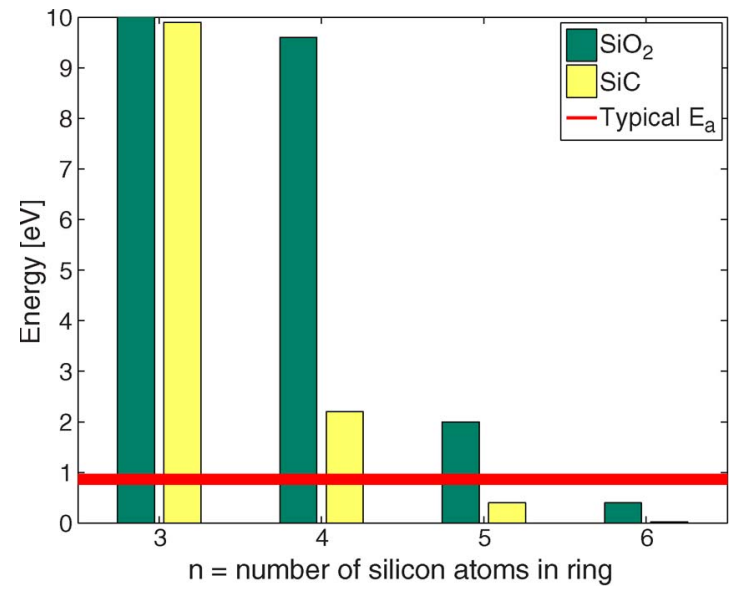

Fig. 3. At what $n$ do $n$-member rings become permeable "holes"? The horizontal red line represents a range of typical $E_{a}$ for $L=0.5 \mu \mathrm{m}$ : The lower bound is $0.79 \mathrm{eV}$ ( $t=2$ months for $\mathrm{SiO}_{2}$ ); the upper bound is $0.94 \mathrm{eV}$ $(t=20$ years for $\mathrm{SiC})$. Note that the activation energies of 0.82 (corresponding to $t=2$ months for $\mathrm{SiC}$ ) and $0.92 \mathrm{eV}$ (corresponding to $t=20$ years for $\mathrm{SiO}_{2}$ ) fall within this range. The green and yellow bars are interior energies for $\mathrm{SiO}_{2}$ and $\mathrm{SiC}$, respectively. For all practical purposes, silica devices are impenetrable to water vapor when $n \leq 5$; for $\mathrm{SiC}, n \leq 4$.

diffusion time of $t=5$ years and length $L=0.5 \mu \mathrm{m}$ in silica, a $10 \%$ increase in $k T$ from 0.026 to $0.0286 \mathrm{eV}$ (an increase in operating temperature from about $27^{\circ} \mathrm{C}$ to $59^{\circ} \mathrm{C}$ ) increases the activation energy from 0.88 to $0.97 \mathrm{eV}$, yet these differences are still smaller than those in $E_{a}$ for different $n$ 's.

Assuming a relatively constant temperature, for both $\mathrm{SiO}_{2}$ and $\mathrm{SiC}$, differences in $E_{a}$ between different $n$ 's are large compared to the smaller-than-0.1-eV difference in $E_{a}$ for varying diffusion times (e.g., from five years to two months). The horizontal diffusion time lines in Fig. 3 give rise to an unambiguous cutoff value for the desired number of ring members. "Holes," or ring sizes responsible for water permeation, can therefore be clearly identified by their number of ring members $n$, as shown in Fig. 3. For example, by Fig. 3, we know that SiC rings no larger than four-member rings are desired in order to prevent water diffusion. Similarly, we see that a-SiO 2 should be composed of rings with five or fewer members. Thus, such plots show at what $n$ rings become permeable pathways for water molecules.

Protective thin films can be fabricated by plasma-enhanced chemical vapor deposition as was shown in [14]. The reliable fabrication of impermeable (no-"hole" pathways) a- $\mathrm{SiO}_{2}$ will require further investigation into the growth kinetics of thin films. Recent work suggests that there exists a weak coupling between the structure of bilayer a- $\mathrm{SiO}_{2}$ films and that of their substrates (e.g., ruthenium in [3] and graphene in [15]). This may provide a platform for growing $\mathrm{a}-\mathrm{SiO}_{2}$ thin films with preferred ring-size distributions.

\section{Concluding Remarks}

Given the results of Fig. 3, we see that the question shifts from the precise evaluation of ring barrier energies (e.g., AM1 versus B3LYP calculations) to the estimates of the fraction and network topology of water-permeable "holes," the latter being defined by the activation energy corresponding to the desired diffusion time and length. Plots such as Fig. 3 yield a definite value of $n$ at which $n$-member rings become holes (i.e., gates for water molecules to diffuse). In a-SiO $\mathrm{Si}_{2}, n$-member rings with $n>5$ are holes; in a-SiC, this condition yields $n>4$. Thus, for all practical purposes, silica devices are impenetrable to water vapor for $n \leq 5$; for silicon carbide devices, $n \leq 4$. In view of these results, we propose that a layer of a nonreactive amorphous-silicon-based compound, composed of rings of $n \leq$ 5 , would serve as a water-vapor-impermeable protective coating on the decadal time scale. Note that the fraction of holes need not be zero; rather, holes must be isolated so that a percolation path for water molecules cannot form.

\section{ACKNOWLEDGMENT}

The authors would like to thank $\mathrm{H}$. He for the helpful discussions.

\section{REFERENCES}

[1] T. Michalske and S. Freiman, "A molecular mechanism for stress corrosion in vitreous silica," J. Amer. Ceram. Soc., vol. 66, no. 4, pp. 284-288, Apr. 1983.

[2] F. Lechenault, C. Rountree, F. Cousin, J. Bouchaud, L. Ponson, and E. Bouchaud, "Evidence of deep water penetration in silica during stress corrosion fracture," Phys. Rev. Lett., vol. 106, no. 16, p. 165 504, Apr. 2011.

[3] L. Lichtenstein, C. Bchner, B. Yang, S. Shaikhutdinov, M. Heyde, M. Sierka, R. Wlodarczyk, J. Sauer, and H.-J. Freund, "The atomic structure of a metal-supported vitreous thin silica film," Angew. Chem. Int. Ed., vol. 51, no. 2, pp. 404-407, Jan. 2012.

[4] S. Kohara and K. Suzuya, "Intermediate-range order in vitreous $\mathrm{SiO}_{2}$ and $\mathrm{GeO}_{2}$," J. Phys. Condens. Matter, vol. 17, no. 5, pp. S77-S86, Feb. 2005.

[5] I. Batyrev, B. Tuttle, D. Fleetwood, R. Schrimpf, L. Tsetseris, and S. Pantelides, "Reactions of water molecules in silica-based network glasses," Phys. Rev. Lett., vol. 100, no. 10, pp. 105 503-1-105 503-4, Mar. 2008.

[6] H. He, M. Deshpande, R. Brown, R. Pandey, and U. Pernisz, "Molecular modeling of water diffusion in amorphous SiC," J. Appl. Phys., vol. 98, no. 2, pp. 023519-1-023519-5, Jul. 2005.

[7] F. Reif, Fundamentals of Statistical and Thermal Physics, 1st ed. New York: McGraw-Hill, 1965, p. 488.

[8] A. Pasquarello and R. Car, "Dynamical charge tensors and infrared spectrum of amorphous $\mathrm{SiO}_{2}$," Phys. Rev. Lett., vol. 79, no. 9, pp. 1766-1769, Sep. 1997.

[9] M. Tomozawa and K. Davis, "Time dependent diffusion coefficient of water into silica glass at low temperatures," Mater. Sci. Eng. A, Struct., vol. 272, no. 1, pp. 114-119, Nov. 1999.

[10] M. J. Frisch, et al., Gaussian 98. Gaussian, Inc., Pittsburgh PA, 1998.

[11] M. Dewar, E. Zoebisch, and E. Healy, "Development and use of quantum mechanical molecular models. 76. AM1: A new general purpose quantum mechanical molecular model," J. Amer. Chem. Soc., vol. 107, no. 13, pp. 3902-3909, Jun. 1985.

[12] A. Becke, "Density-functional thermochemistry. III. The role of exact exchange," J. Chem. Phys., vol. 98, no. 7, pp. 5648-5652, Apr. 1993.

[13] C. Lee, W. Yang, and R. Parr, "Development of the Colle-Salvetti correlation-energy formula into a functional of the electron density," Phys. Rev. B, vol. 37, no. 2, pp. 785-789, Jan. 1988.

[14] L. Zambov, K. Weidner, V. Shamamian, R. Camilletti, U. Pernisz, M. Loboda, G. Cerny, D. Gidley, H. Peng, and R. Vallery, "Advanced chemical vapor deposition silicon carbide barrier technology for ultralow permeability applications," J. Vac. Sci. Technol. A, vol. 24, no. 5, pp. 1706-1713, Sep. 2006.

[15] P. Huang, S. Kurasch, A. Srivastava, V. Skakalova, J. Kotakoski, A. V. Krasheninnikov, R. Hovden, Q. Mao, J. C. Meyer, J. Smet, D. A. Muller, and U. Kaiser, "Direct imaging of a two-dimensional silica glass on graphene,” Nano Lett., vol. 12, no. 2, pp. 1081-1086, Feb. 2012. 